Research Article

\title{
Interactive Teaching Mode of College English Based on Internet of Things
}

\author{
Ting Pu $\mathbb{D}^{1}{ }^{1}$ Xing Liu, ${ }^{2}$ and Wenjing Hao ${ }^{1}$ \\ ${ }^{1}$ Department of Foreign Languages, Sichuan University of Communications, Chengdu 610000, Sichuan, China \\ ${ }^{2}$ School of Foreign Languages, Sichuan Normal University, Chengdu 610000, Sichuan, China \\ Correspondence should be addressed to Ting Pu; puting-eteacher@scmc.edu.cn
}

Received 19 July 2021; Revised 27 August 2021; Accepted 7 September 2021; Published 20 September 2021

Academic Editor: Sang-Bing Tsai

Copyright $\odot 2021$ Ting Pu et al. This is an open access article distributed under the Creative Commons Attribution License, which permits unrestricted use, distribution, and reproduction in any medium, provided the original work is properly cited.

In terms of English teaching in China, English teaching mode, there are some drawbacks. The interaction between teachers and students is very insufficient, which cannot fully mobilize the enthusiasm of students. The purpose of this study is to explore a new way for English teaching mode, hoping to explore better English teaching methods and improve students' English performance. This paper focuses on the characteristics of College English (CE) interactive teaching mode based on the Internet of Things technology, discusses the practical application of the Internet of Things technology in College English interactive teaching, and makes a simple research on the interactive teaching mode of College English based on the Internet of Things (IoT). Students from two natural classes in our city were selected as the research objects and divided into an experimental group and control group. The overall English levels of the two classes were similar, and the study lasted for a semester. Research tools include questionnaires, writing tests, and interviews. Statistical software was used to analyze the data in detail. Before and after the experiment, the test results of the experimental class and the control class were tested by independent sample $t$-test and paired sample $t$-test. The experimental results show that the College English interactive teaching mode based on the Internet of Things is welcomed by students, and the experimental effect is significant. The overall average score of the experimental group increased by 13.6 points, while that of the control group increased by 4.2 points. The gap between the two groups was very obvious. As far as the scores of each part of the English test paper are concerned, the scores of each part of the experimental group are also higher than those of the control group.

\section{Introduction}

1.1. Background and Significance. Teachers must cultivate the ability of pupils to apply at the same time; they must achieve the overall development of hearing, speech, reading, and writing. Interactive teaching language function refers to the process of communication between teachers and students and between students and students. In this process, students gain knowledge and exercise their abilities. The teaching model is transformed from an open, passive learning into an active learning process, which will help them form a new learning structure model. Interactive teaching mode is a unified process of mutual influence and restriction. Through the information exchange and interaction, mutual understanding and teaching reciprocity are formed. Therefore, interactive teaching has attracted increasing attention. Interactive technology refers to a technology that transforms a traditional space into a novel interactive space through an advanced video motion capture system and a mature three-dimensional game engine. Interactive technology breaks the traditional static space model and integrates the audience into a part of the space.

1.2. Related Work. The Internet of Things is considered the future Internet, which includes billions of intelligent communication "things." Li S systematically reviews the definition, architecture, basic technology, and applications of the Internet of Things. Al-Fuqaha A first provides a brief overview of the Internet of Things and then describes some technical details related to the technology, protocol, and applications of the Internet of Things. Sicari $S$ et al. mainly put forward 
challenges and solutions for the security problems in the IoT and put forward practical suggestions [1].

The difficulty of graduates' employment has always been a prominent problem in China. The international financial crisis has made the current employment situation more severe. Zhang Y analyzed in detail the current status of entrepreneurship in our country's universities, combined with the entrepreneurial education model, and innovatively proposed a plan [2]. Functional dependence and the association rule are two important concepts in the database system and are also difficult points in teaching the introduction to the database system. Functional dependence is the theoretical basis for the normalisation of a relative shape, which reflects a relationship of limitation between characteristics in relation. Extracting connection rules is an important research content in data analysis. Yao makes full use of the relationship between functional dependence sets of elements and proposes an algorithm to extract association rules based on functional armafd, which enriches the teaching concept of functional dependence. In addition, the application of data dependence to the extraction of correlation rules is introduced. They will further study the interconnection rules and data dependence [3].

\subsection{Innovation}

(1) Since teachers can upload their online software and exercises collected on the IoT to the IoT, a large collection of courses in terms of things, students can choose their own English learning resources according to their own circumstances and progress.

(2) The selection of teaching materials is often guided by teachers due to the differences in learning ability and learning process of each student in a class. The teaching materials will gradually be dominated by students, and many extracurricular learning opportunities will remedy the lack of class time, and they will get more time to interact with teachers.

\section{CE Interactive Teaching Mode Based on the IoT}

2.1. Limitations of Interactive Teaching Mode. First, it seeks only formal interaction. This kind of interactive teaching is usually called "programmed interaction between teacher and students." Although teachers are well designed, this knowledge transfer can largely predict students' reactions. The interaction depth is not enough, and most of them are shallow interactions [4]. Because interactive technology requires a way of displaying images, interactive technology is now mainly divided into two parts: projection interactive technology and video interactive technology. Therefore, how to deepen the level of interaction and fully mobilize pupils' abilities is a problem that must be solved in multidimensional interactive teaching. The ground interactive technology is an interactive technology that displays the display effect picture on the ground, and the ground interactive technology is generally realized through the projection interactive technology.

Secondly, although teachers have achieved interaction in the teaching process and fully mobilized the subjective initiative of students, only a few students' interest has been aroused, and most students are in a depressed, passive, and stagnant learning state. There are differences in the amount of knowledge, personality characteristics, psychological characteristics, family status, intelligence, and so on. It is precisely because of the differences of these interactive resources that teachers can not enjoy the monopoly of these resources in the classroom, which will eventually lead to polarization of learning results [5].

Third, some teachers do not really understand the nature of interactive teaching, mistakenly equate interactive teaching with rigid teaching activities in the classroom, or understand the interaction as "integration" with students, and think that students' discussion activities and classroom game activities are interactive teaching [6]. So, the basic knowledge and skills of primary school students do not meet the standard. On the contrary, it is easy to cause many students to develop a bad habit of disrespect for classroom discipline and freedom.

Fourthly, the interaction is mainly focused on teachers. Teachers are still the initiators and controllers of classroom interactive teaching, playing the role of "judge" and "referee." The illusion to students is that they must interact with teachers as much as possible in class, and the interaction with students is not important [7]. In addition, because the number of students is much higher than that of teachers, the interaction ratio between one teacher and multiple students is totally different. This will inevitably lead to a single form of interactive teaching, to a large extent, to prevent students' initiative and creativity.

Fifthly, in the teaching content, there are many cognitive interactions. The topics discussed are all learning contents, but there is little interaction in emotion and values. This situation is not interactive teaching. This is an important factor, and this is also a common problem in college English classes.

Sixth, the content value of cooperative learning is not high, and the lack of necessary preparation before group cooperation and the provision of discussion time are unreasonable, which will cause students to lose interest in group topics, and the cooperation between students is not active enough. Such group interaction cannot achieve a good teaching mode and will eventually waste classroom teaching time [8]. The application of interactive technology in homes, schools, and playgrounds is mainly divided into two categories: one is children's game products and the other is children's playgrounds.

2.2. Types of Interactive Teaching. Each teaching process has its own unique behavior orientation. The behavior orientation of interactive teaching can be divided into three types: one is the interaction between teachers and students; the other is the interaction between students and students; the third is the self interaction of students. 
2.2.1. Teacher-Student Interaction. On the issue of teacherstudent relationship, people have been more concerned about the attitude and the teacher's role in this regard, hoping to create a harmonious relationship. However, this does not mean that students, as a person with independent personality and self-construction abilities, play their own role. In the interactive classroom, the teacher is not an initiator, but a promoter, and the situation of teacher and student is equal [9]. Through this interactive form, students' wisdom and thoughts can be shared and inspired, and students' knowledge structure can be established to improve students' writing ability and teachers' teaching ability.

2.2.2. Interaction among Students. According to the theory of interactive teaching, only interpersonal interaction can produce a beneficial learning result. This shows the important role of interaction in learning [10]. However, the traditional teaching method has only teacher-student interaction, which is considered negative or irrelevant. Especially in written teaching in China, this situation is more common. In general, the teacher decides, examines the subject, selects the material, and designs the layout of the article and then the student continues writing; the student's composition is always completed in her own circle of thought. The teacher then corrected the pupils'compositions, and the pupils improved their learning in response to criticism.

2.2.3. Students' Interaction. Self-interaction refers to the internal communication. In this process, students are the only object of communication. Emotional communication and language expression are complex psychological activities. In this sense, self-interaction mode is the ultimate goal [11].

\subsection{Teaching Mode Based on the IoT. The Internet of Things} is an extension and expansion network based on the Internet. A large network is formed to realize the interconnection of people, machines, and things anytime and anywhere. The Internet of Things (referred to as IOT) refers to real-time collection of information sensors, radio frequency identification technology, global positioning system, infrared sensors, laser scanners, and other devices and technologies that need to be monitored, connected, and interacted. Objects or processes, collecting various required information such as sound, light, heat, electricity, mechanics, chemistry, biology, and location.

Through various possible network accesses, realize the ubiquitous connection between things and people, and realize the intelligent perception, recognition, and management of things and processes. The Internet of Things is an information carrier based on the Internet and traditional telecommunications networks. The application field of the Internet of Things involves all aspects. The application in the field of infrastructure such as industry, agriculture, environment, transportation, logistics, and security has effectively promoted the intelligent development of these areas, making the limited resources more reasonably used and allocated, thereby improving the efficiency and benefit of the industry.

2.3.1. The Combination of English Teaching and the IoT. The combination of the IoT and English content can improve students' English proficiency at the fastest speed. Through all kinds of possible network access, realize the ubiquitous connection between things and things and things and people, and realize the intelligent perception, recognition, and management of things and processes. The Internet of Things is an information carrier based on the Internet and traditional telecommunications networks. We introduce the IoT in the original English learning process, such as listening to lessons, taking notes, and writing, to guide students to actively use tools for extracurricular entertainment and communication [12, 13]. Radio frequency identification technology is a simple wireless system consisting of an interrogator (or reader) and many transponders (or tags). It transmits radio frequency information to the reader through an antenna, and the reader is a device that reads the information. Because sensors give ordinary objects a new life, they have their own data transmission channels, storage functions, operating systems, and specialized applications, thus forming a large sensor network. This allows the Internet of Things to monitor and protect people through objects. The use of IoT can provide a very strong internal incentive for students. The Internet business of things is just beginning. One of the core concepts of cloud computing is to continuously improve the processing capacity of the "cloud" and continuously reduce the processing burden of the user terminal and finally simplify it into a simple input and output device and enjoy the powerful computing processing capabilities of the "cloud" on demand. In these circumstances, if you want to find good work in the future, you must rely on English, the Internet, and the Internet for the things that have developed in recent years [14]. The IoT places English in an international environment. Students can also learn according to their own comfortable rhythm. The Internet of Things is an extended and expanded network based on the Internet. It is a large network formed by combining various information sensing devices on the network, realizing the interconnection of people, machines, and things anytime and anywhere.

\subsubsection{Six Functions of the IoT in English Teaching}

(1) Information retrieval and acceptance: they include some language activities such as a large virtual library, and these include activities just like looking for and obtaining information in the library. According to the principle of information retrieval technology, the information to be stored includes not only the original document data, but also pictures, videos, and audio. First, the original information must be converted into computer language and stored in the database; otherwise it cannot be machined. 
(2) Disclosure and provision of information: publishing blogs written by students in English on the Internet can play a role in communication and mutual learning.

(3) Internet communication: this mainly refers to the exchange activities through e-mail or chat room on the Internet of Things (QQ: e-mail chat with foreign friends).

(4) Cooperation and learning: this mainly involves cooperation between two distant pupils or classes, i.e., to participate in some English activities together. We can communicate with foreign teaching classes in the classroom and create websites to guide students on both sides to study the history, geography, climate, and environment of each other's cities. Through this way of communication, students can gain more extracurricular knowledge.

(5) WeChat group learning: in the initial stage, create a class WeChat group. In addition to delivering important information in Chinese and English, the messages in the WeChat group should be delivered in English [15]. The biggest advantage of creating a class-based WeChat group is that it can let students learn English in entertainment and form a pleasant English learning atmosphere.

(6) Internet of Things can provide a good environment for learning English. At this stage, many students are Internet fans. Due to the characteristics of the modern college students, the student may be an appropriate network boot and the network will combine learning English, so students become addicted to the Internet enthusiasts learning English.

2.4. Smart Sensors. Network communication technology has also seen a rapid development. Digital sensors are with low intelligence. Therefore, intelligent, structured, and networked sensors have become the development direction of new sensors, and intelligent sensors are thus born. Unlike the analog sensor and the digital sensor, the smart sensor has the function of processing information. For example, in industrial production, the use of analog sensors and digital sensors cannot quickly measure and control certain product quality indicators (such as composition, surface finish, colour, taste, and viscosity) [16, 17]. However, if it is an intelligent sensor, it can quickly measure some quantities (such as temperature, pressure, and processes that are functionally related to product quality indicators during production and processing). The microprocessor can calculate, store, and process the data measured by the sensor [18]. It can also adjust the sensor through the feedback loop. It is the core of the intelligent sensor. Sensor technology is an important part of realizing, testing, and automatic control. In the test system, it is used as an instrument positioning, and its main feature is that it can accurately transmit and detect a certain form of information and convert it into another form of information.
The smart sensor consists of about seven parts, as shown in Figure 1. The sensing unit detects the information from the outside, and through signal regulation, it can process the signal and prepare for the analog-to-digital conversion of the data [19]. Application algorithm refers to the material and software of the application layer, to convert the collected data into user-defined units, data classification, signal analysis and processing, alarm monitoring, recording time stamps in memory, running a control loop, or making the result closer to the measurement point; the algorithm provides "intelligence" for the intelligent sensors. Data storage is used to store sensor identification and structure information, time mark data, scale data, and user display settings. Almost all or part of the sensor files are stored in the corresponding sensor storage units, such as historical users and manufacturers, user manuals, and installation process. The local user interface is a standard component through which the user's language and terminology can be corrected and displayed in front of the end user. The remote user interface is connected to the end user and can be used for calibration, diagnosis, data acquisition, and daily monitoring.

\section{Experimental Research on Interactive Teaching Mode of CE Based on the IoT}

3.1. Research Object.

(1) Questionnaire method: through the questionnaire survey of college professors at five different levels of schools in our province, we can understand and analyze the limitations of current English teaching in college, the attitude of college professors, the understanding, and the use of the computer network and submit proposals for designing the interactive method of teaching the Internet of Things in the next step.

(2) Experimental analysis: this study selected two natural classes in our city as the research object. These students have been with the same English teacher since their freshman year. The students in the two classes have a stable English foundation. Therefore, the two classes are selected as the objects of the comparative experiment, and the experiment will be carried out by sophomore. The two classes are sophomore (5) and sophomore (6). Sophomore (5) is selected as the experimental class, and sophomore (6) is the control class. Before the experiment, it is necessary to implement a pretest for the students in the two classes. The average scores of the two classes are almost the same.

3.2. Experimental Steps. The experiment uses literature review methods and comparative analysis to carry out the experiment. Experiments and questionnaires are the research tools used before the experiment. The two classes were tested in early September 2018, and the test papers were the English test questions of the final freshman examination of our university from 2017 to 2018 . The paper consists of five parts. The main problem is the type of hearing, multiple choices, 


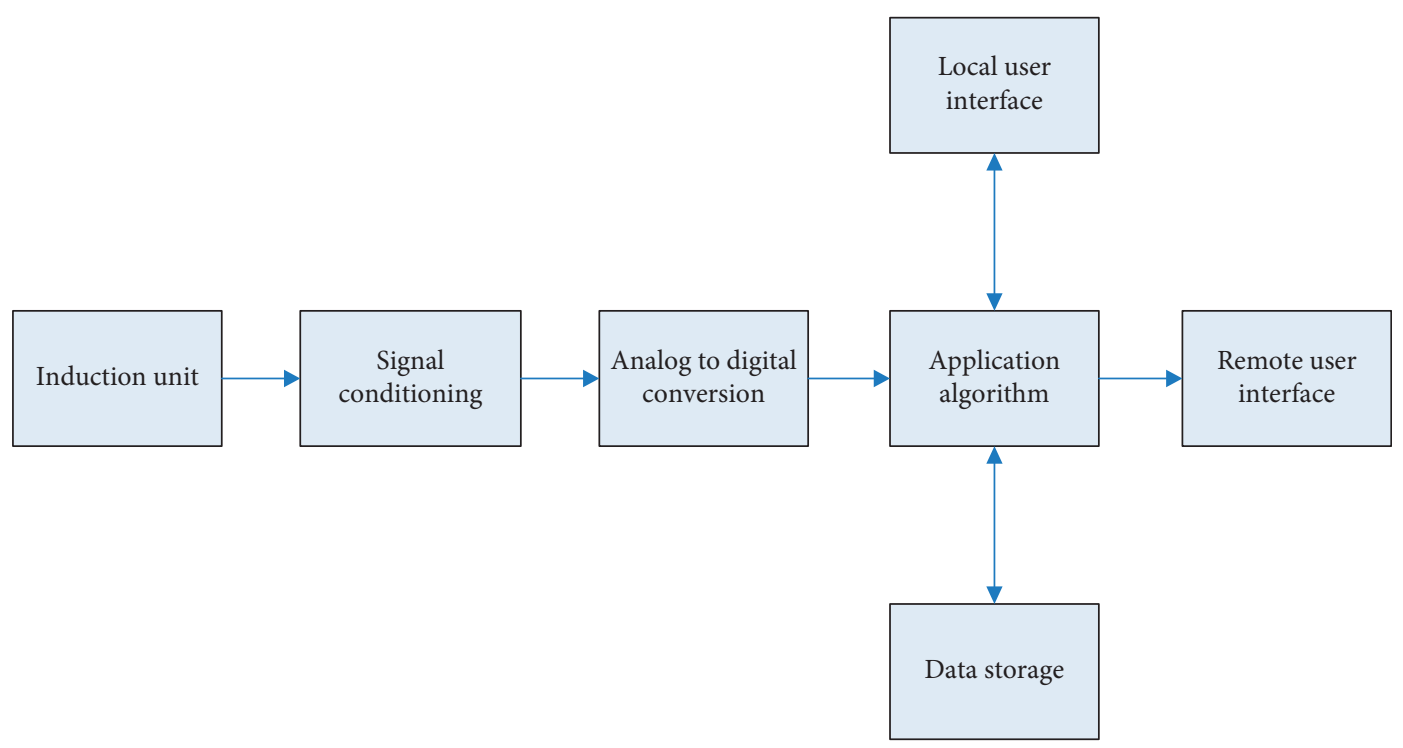

FIGURE 1: Basic structure unit of intelligent sensor.

cloze, reading comprehension, and written expression. The total score is 100. Finally, this paper makes detailed statistics of the scores of the two classes in each part of the test, aiming to make a comparative analysis of the students' listening, writing, and reading achievements of the two classes.

3.3. Welch $t$-Test. All experimental data in this section are analyzed by statistical software. Student's $t$-test is used when the variance of two populations is unknown but equal. When the sample size and variance of two populations are not equal, Welch $t$-test can be used:

$$
t=\frac{X_{A}-X_{B}}{\left(S_{A}^{2} / N_{A}\right)+\left(S_{B}^{2} / N_{B}\right)},
$$

where $X_{A}$ and $X_{B}$ are the sample mean values in sets $A$ and $B$. In Welch $t$-test, the original hypothesis and alternative hypothesis were

$$
\begin{gathered}
H_{0}: \mu_{A}=\mu_{B}, \\
H_{1}: \mu_{A} \neq \mu_{B} .
\end{gathered}
$$

The critical value $C$ needs to be calculated according to the significance level $\alpha$ and the degree of freedom $V$ to determine the rejection region. When $|t| \geq C$, it is rejected; otherwise $H_{0}$ is accepted.

$$
t=\frac{\left(S_{A}^{2} / N_{A}\right)+\left(S_{B}^{2} / N_{B}\right)}{\left(\left(S_{A}^{2} / N_{A}\right)^{2} /\left(N_{A}-1\right)\right)+\left(\left(S_{B}^{2} / N_{B}\right)^{2} /\left(N_{B}-1\right)\right)} .
$$

\section{Experimental Analysis of CE Interactive Teaching Mode Based on the IoT}

4.1. Teaching Mode Based on the IoT. University teachers can build a website of auxiliary teaching through network technology and their own teaching ideas, divided into several different topics, such as courseware download and online testing. In the courseware download center, students can download their own teaching courseware anytime and anywhere according to their learning progress, to realize personalized learning to the maximum extent. In the online test column, teachers upload various types of test questions, which are classified according to the degree of difficulty. Students can choose different types of test questions according to their English level. Table 1 shows the role of interactive teaching mode based on the IoT.

As shown in Table 1 , the students are very satisfied with the interactive teaching mode. $80 \%$ of the students think that the teaching mode is very effective.

First of all, the teacher establishes a WeChat group with the class as the unit and elects a manager to maintain the daily affairs in the WeChat group. Secondly, teachers should formulate a set of group rules according to students' requirements or actual situation. For example, in the WeChat group, English must be used for communication. In addition, the biggest advantage of the WeChat group is that it can create a positive and relaxed learning atmosphere for students. Then they can learn English with an entertainment attitude. Figure 2 shows students' knowledge in class after the experiment.

As shown in Figure 2, the situation of students' classroom knowledge has changed. After receiving the teaching, $57 \%$ of the students think they have mastered most of the English classroom knowledge, and 33\% of the students think that they have mastered all English knowledge, and a small part of the students think they have mastered a small part of the knowledge.

4.2. Test Average Scores of the Two Classes before and after the Experiment. The experimental results of the two classes are not accurate enough to avoid the experimental results. And the number of students in these two groups is the same, and the consistency of the experimental samples should be kept as much as possible. Table 2 is a comparison of basic information of students. 
TABLE 1: The function of interactive teaching mode.

\begin{tabular}{lcc}
\hline & Number of students & Proportion (\%) \\
\hline Effective & 112 & 80 \\
Not obvious & 11 & 7.8 \\
Have no feelings & 8 & 5.7 \\
Invalid & 9 & 6.5 \\
\hline
\end{tabular}

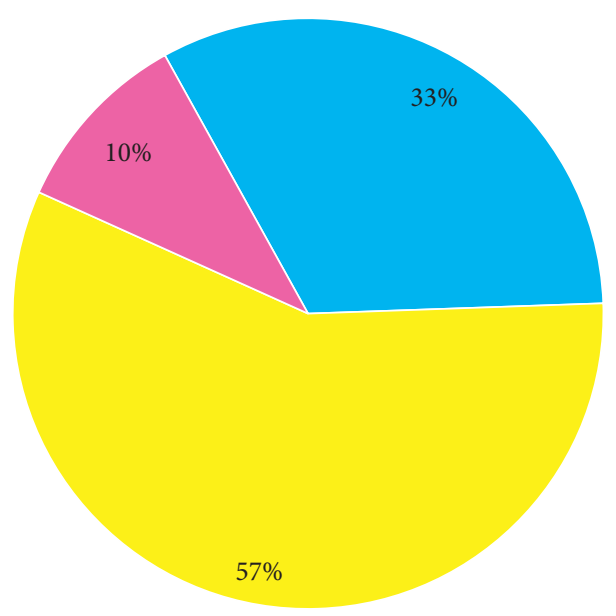

- All

1 Most

- Smaller part

Figure 2: Students' mastery of knowledge in class.

TABLE 2: Two classes of student's basic situation questionnaire.

\begin{tabular}{lccc}
\hline Project & Category & Number of people & Percentage \\
\hline \multirow{2}{*}{ Gender } & Man & 96 & 58 \\
& Woman & 68 & 42 \\
\hline \multirow{2}{*}{ Class category } & Experimental class & 82 & 50 \\
& Control class & 82 & 50 \\
\hline
\end{tabular}

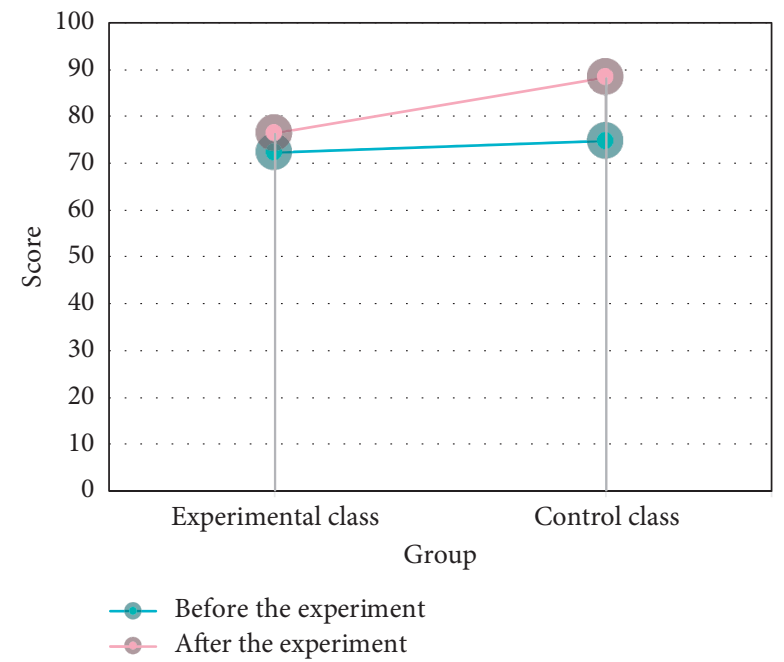

Figure 3: Changes in student average scores. 
The average scores of the two classes before and after the experiment are shown in Figure 3.

Analysis of the data in the chart shows that, after the experiment, the average English scores of the two classes have increased to varying degrees. Compared with the pretest, the average score of the posttest has increased by 13.6 points, and compared with the pretest, the average score of the control class increased by 4.2 points.

\subsection{Comparative Analysis of the Scores of Each Part of the} Experimental Group before and after the Experiment. Under the background of the new curriculum reform, the demand for updating College English teaching mode is increasing to strengthen the construction of interactive teaching mode. Teachers' recognition of interactive teaching has been improved. In addition, the teachers interviewed all mentioned that today's students generally have a smart phone, and they also have computers at home. Even if they do not, the school's computer room can start the borrowing scheme to meet the students' learning needs after class. The hardware facilities for experimental teaching are not a problem. The English test paper is divided into five parts: listening $(\mathrm{H})$, single choice $(\mathrm{s})$, cloze (c), reading comprehension (R), and written expression (W). The scores of each part of the experimental group before and after the experiment are compared as shown in Figure 4.

The scores of all parts of the experimental class students have increased; the most obvious is that the scores of cloze and reading comprehension have increased the most. In the test before the experiment, the average listening score of the experimental class is 10.3, and after the experiment, the average score of listening has increased to 12.9. By using the interactive teaching method and the overall method of hearing and writing, pupil hearing has improved significantly and the teaching result has improved significantly. Prior to the experiment, the average score of pupils' written expressions in the experimental class was 15.6 and the average score of pupils' written expressions after the experiment was 17.1. The score in the unit of one selection was also increased from 12.6 before the test to 14.4 .

4.4. Ability Analysis and Comparison of Teaching Mode. The content of the interview mainly focuses on students' selfevaluation, cognition, cooperation, and problem-solving ability. Figure 5 shows the ability analysis and comparison of CE interactive teaching mode based on the IoT.

From the figure, we can know that the experimental groups are better than the control group in self-study, cooperation, and problem-solving ability under the new teaching mode environment. College English interactive teaching provides a new learning method. Therefore, all students can participate in learning and stimulate students' learning motivation and belonging in learning and promote the friendship among students. Therefore, the interactive method is bound to be welcomed by students.

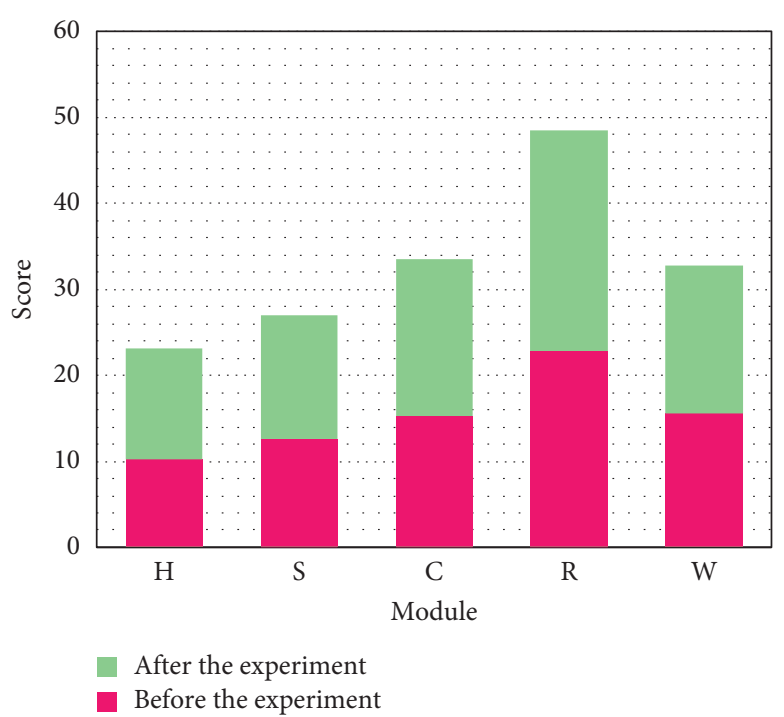

Figure 4: Before and after the experiment, the scores of each part of the experimental group.

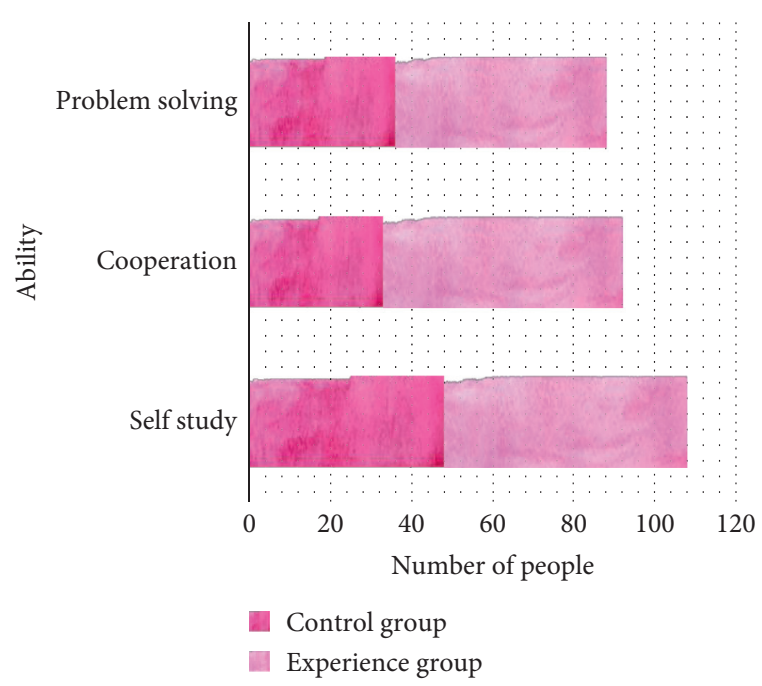

FIgURE 5: Capability analysis and comparison of CE interactive teaching mode based on the IoT.

\section{Conclusions}

Although this study has its inevitable limitations, under the guidance of the interactive teaching methods, it can improve students' writing level. This study can be used as a supplement to interactive case analysis to help develop interactive cases. This study can also serve as an example for College English teachers. Most importantly, it can also provide materials for students and teachers to further study English teaching. It can be seen that the interactive teaching method is effective for the improvement of performance.

Both teachers and students must understand autonomous learning correctly. As a teacher, its leading role cannot be ignored; otherwise students will lose control of learning, because modern educational technology is not only a teaching 
tool but also an entertainment tool. If used improperly, it may backfire. Students should not regard "independent learning" as "free learning," nor should they believe that they are not restricted by teachers and can do whatever they want. On the contrary, students should realize that "successful learners usually have strong self-management abilities." They not only have the skills and strategies to participate in various learning activities and solve various learning difficulties, but also can choose their own learning methods according to the specific learning tasks. This is the real self-learning. Only in this way can the Internet of Things assisted learning achieve the desired effect.

Teachers can upload the online materials and exercises they collected to the IoT through the upload function. Students can choose their own English learning resources. This learning method can significantly improve student achievement. Book selection is often guided by the teacher. Because it is different in the same class, with each student's learning ability and learning progress, the book does not fully meet the class to learn English. A large number of afterschool learning opportunities make up for the lack of classroom books, and students can have more time to interact in class.

\section{Data Availability}

The data underlying the results presented in the study are available within the manuscript.

\section{Conflicts of Interest}

The authors declare that they have no conflicts of interest.

\section{References}

[1] S. Sicari, A. Rizzardi, L. A. Grieco, and A. Coen-Porisini, "Security, privacy and trust in Internet of Things: the road ahead," Computer Networks, vol. 76, no. 15, pp. 146-164, 2015.

[2] Y. Zhang, "Study on college teaching mode of startups education in perspective of innovation ability," Open Journal of Leadership, vol. 4, no. 1, pp. 30-34, 2015.

[3] Y. Yao, "Application of flipped classroom teaching mode based on MOOC in modern educational technology teaching," Journal of Computational and Theoretical Nanoscience, vol. 14, no. 2, pp. 1075-1078, 2017.

[4] R. Shah and Y. Wang, "Cloud Things Construction - the integration of Internet of things and cloud computing," Future Generation Computer Systems, vol. 56, no. C, pp. 684-700, 2016.

[5] J. H. Ziegeldorf, O. G. Morchon, and K. Wehrle, "Privacy in the internet of things: threats and challenges," Security and Communication Networks, vol. 7, no. 12, pp. 2728-2742, 2015.

[6] L. Yancong, Y. Jiachen, L. Zhihan, W. Wei, and H. Song, "A self-assessment stereo capture model applicable to the internet of things," Sensors, vol. 15, no. 8, pp. 20925-20944, 2015.

[7] M. A. Razzaque, M. Milojevic-Jevric, A. Palade, and S. Clarke, "Middleware for internet of things: a survey," IEEE Internet of Things Journal, vol. 3, no. 1, pp. 70-95, 2017.

[8] J. Granjal, E. Monteiro, and J. Sa Silva, "Security for the internet of things: a survey of existing protocols and open research issues," IEEE Communications Surveys \& Tutorials, vol. 17, no. 3, pp. 1294-1312, 2015.
[9] J. Lin, W. Yu, N. Zhang, X. Yang, H. Zhang, and W. Zhao, “A survey on internet of things: architecture, enabling technologies, security and privacy, and applications," IEEE Internet of Things Journal, vol. 4, no. 5, pp. 1125-1142, 2017.

[10] O. Bello and S. Zeadally, "Intelligent device-to-device communication in the internet of things," IEEE Systems Journal, vol. 10, no. 3, pp. 1172-1182, 2016.

[11] G. Liu, "Application of mind mapping method in college English vocabulary teaching," Open Journal of Modern Linguistics, vol. 06, no. 3, pp. 202-206, 2016.

[12] A. V. Dastjerdi and R. Buyya, "Fog computing: helping the internet of things realize its potential," Computer, vol. 49, no. 8, pp. 112-116, 2016.

[13] R. M. Dijkman, B. Sprenkels, T. Peeters, and A. Janssen, "Business models for the internet of things," International Journal of Information Management, vol. 35, no. 6, pp. 672-678, 2015.

[14] J. Mineraud, O. Mazhelis, X. Su, and S. Tarkoma, "A gap analysis of Internet-of-Things platforms," Computer Communications, vol. 89-90, no. 1, pp. 5-16, 2016.

[15] A. Aijaz and A. H. Aghvami, "Cognitive machine-to-machine communications for internet-of-things: a protocol stack perspective," IEEE Internet of Things Journal, vol. 2, no. 2, pp. 103-112, 2015.

[16] S. N. Mohanty, E. L. Lydia, M. Elhoseny, M. M. G. Al Otaibi, and K. Shankar, "Deep learning with LSTM based distributed data mining model for energy efficient wireless sensor networks," Physical Communication, vol. 40, Article ID 101097, 2020.

[17] K. Visvam Devadoss Ambeth, "Human security from death defying gases using an intelligent sensor system," Sensing and Bio-Sensing Research, vol. 7, no. C, pp. 107-114, 2016.

[18] W. Elsayed, M. Elhoseny, S. Sabbeh, and A. Riad, "Selfmaintenance model for wireless sensor networks," Computers \& Electrical Engineering, vol. 70, pp. 799-812, 2018.

[19] E. Schaefer, "Artificial Nose Sniffs out Gas Intelligent sensor creates a database of gas "fingerprints", Elektor Electronics Worldwide, no. 3, pp. 44-47, 2017. 